\title{
Menatetrenone versus alfacalcidol in the treatment of Chinese postmenopausal women with osteoporosis: a multicenter, randomized, double-blinded, double- dummy, positive drug-controlled clinical trial
}

\author{
This article was published in the following Dove Press journal: \\ Clinical Interventions in Aging \\ 8 January 2014 \\ Number of times this article has been viewed
}

\author{
Yan Jiang ${ }^{1, *}$ \\ Zhen-Lin Zhang 2 ,* \\ Zhong-Lan Zhang ${ }^{3}$ \\ Han-Min Zhu ${ }^{4}$ \\ Yi-Yong $\mathrm{Wu}^{5}$ \\ Qun Cheng ${ }^{4}$ \\ Feng-Li Wu \\ Xiao-Ping Xing' \\ Jian-Li Liu ${ }^{3}$ \\ Wei Yu ${ }^{6}$ \\ Xun-Wu Meng' \\ 'Department of Endocrinology, Key Laboratory \\ of Endocrinology, Peking Union Medical College \\ Hospital, Chinese Academy of Medical Sciences, \\ Beijing, ${ }^{2}$ Metabolic Bone Disease and Genetic \\ Research Unit, Department of Osteoporosis \\ and Bone Disease, Shanghai Jiao Tong University \\ Affiliated Sixth People's Hospital, Shanghai, \\ ${ }^{3}$ Department of Gynecology and Obstetrics, \\ General Hospital of the People's Liberation Army, \\ Beijing, ${ }^{4}$ Department of Geriatrics, Shanghai \\ Huadong Hospital, Shanghai, ${ }^{5}$ Department of \\ Gynecology and Obstetrics, Beijing Hospital, \\ Ministry of Public Health, Beijing, ${ }^{6}$ Department of \\ Radiology, Peking Union Medical College Hospital, \\ Chinese Academy of Medical Sciences, Beijing, \\ People's Republic of China \\ *These authors contributed equally to this work
}

Correspondence: Xiao-Ping Xing

Department of Endocrinology, Key Laboratory of Endocrinology, Ministry of Health, Peking Union Medical College Hospital, Chinese Academy of Medical Sciences, Beijing 100730 , People's Republic of China

Tel +86 1069155084

Fax $+86106915 \quad 1552$

Email xingxp2006@I26.com

Jian-Li Liu

Department of Gynecology and Obstetrics, General Hospital of the People's Liberation Army, Beijing 100853, People's Republic of China

Email1316167397@qq.com
Objective: To evaluate whether the efficacy and safety of menatetrenone for the treatment of osteoporosis is noninferior to alfacalcidol in Chinese postmenopausal women.

Method: This multicenter, randomized, double-blinded, double-dummy, noninferiority, positive drug-controlled clinical trial was conducted in five Chinese sites. Eligible Chinese women with postmenopausal osteoporosis $(\mathrm{N}=236)$ were randomized to Group M or Group A and received menatetrenone $45 \mathrm{mg}$ /day or alfacalcidol $0.5 \mu \mathrm{g}$ /day, respectively, for 1 year. Additionally, all patients received calcium $500 \mathrm{mg}$ /day. Posttreatment bone mineral density (BMD), new fracture onsets, and serum osteocalcin (OC) and undercarboxylated OC (ucOC) levels were compared with the baseline value in patients of both groups.

Results: A total of 213 patients (90.3\%) completed the study. After 1 year of treatment, BMD among patients in Group M significantly increased from baseline by $1.2 \%$ and $2.7 \%$ at the lumbar spine and trochanter, respectively $(P<0.001)$; and the percentage increase of BMD in Group A was $2.2 \%$ and $1.8 \%$, respectively $(P<0.001)$. No difference was observed between groups. There were no changes in femoral neck BMD in both groups. Two patients $(1.9 \%, 2 / 108)$ in Group M and four patients $(3.8 \%, 4 / 105)$ in Group A had new fracture onsets $(P>0.05)$. In Group M, OC and ucOC decreased from baseline by $38.7 \%$ and $82.3 \%$, respectively $(P<0.001)$. In Group A, OC and ucOC decreased by $25.8 \%$ and $34.8 \%$, respectively $(P<0.001)$. Decreases in serum $\mathrm{OC}$ and ucOC were more obvious in Group M than in Group A $(P<0.001)$. The safety profile of menatetrenone was similar to alfacalcidol.

Conclusion: Menatetrenone is an effective and safe choice in the treatment of postmenopausal osteoporosis in Chinese women.

Keywords: menatetrenone, alfacalcidol, postmenopausal, osteoporosis, bone mineral density, undercarboxylated osteocalcin

\section{Introduction}

Osteoporosis is characterized by decreased bone strength, and it leads to increased bone fragility and susceptibility to fractures. ${ }^{1}$ Postmenopausal osteoporosis and associated fractures are major public health problems across the world, especially in the People's Republic of China. Osteoporosis is caused by an imbalance between bone tissue formation by osteoblasts and resorption by osteoclasts. ${ }^{2}$

Osteoporosis may be prevented or treated in its early stages using basic approaches (calcium, vitamin D, diet, exercise, and smoking cessation). ${ }^{1} \mathrm{Sev}-$ eral antiosteoporotic drugs have been used widely for its more severe stages, and 
they have demonstrated efficacy in increasing bone mineral density (BMD) and decreasing fracture incidences. Other drugs such as bisphosphonates, ${ }^{3}$ raloxifene, ${ }^{4}$ estrogen/progestin therapy, ${ }^{5}$ and denosumab ${ }^{6}$ have also been found to be effective against osteoporosis.

Menatetrenone (vitamin K2) is known to be a cofactor of $\gamma$-carboxylase, which converts glutamic acid residues in the osteocalcin (OC) molecule to $\gamma$-carboxyglutamic acid, and it is therefore essential for $\gamma$-carboxylation of OC. ${ }^{7-9}$ Low vitamin $\mathrm{K}$ consumption is associated with a higher risk of hip fracture among older women and men. ${ }^{10-13}$ A metaanalysis ${ }^{14}$ has revealed that menatetrenone therapy decreases new vertebral fractures and possibly reduces long bone fractures. Menatetrenone has been approved in Japan in 1995 for treatment of postmenopausal osteoporosis; since then, it has been used widely in that country. ${ }^{15}$

Hence, available published data are mostly obtained from a Japanese population, which may have a different pattern of risk factors (diet, exercise, and genetics) for the disease than a Chinese population. The efficacy and tolerance of menatetrenone in Chinese postmenopausal osteoporotic women has not been previously investigated. This 1-year study was therefore designed to evaluate the efficacy and safety of menatetrenone for the treatment of osteoporosis in Chinese postmenopausal women in comparison with alfacalcidol treatment. Since the study involved osteoporotic women, a placebo control was not used, and alfacalcidol was chosen as a positive drug control to demonstrate the noninferiority of these two drugs.

\section{Patients and methods}

\section{Patients}

The study was approved by the State Food and Drug Administration of China (2004L03447). This study protocol was evaluated by the ethics committee of each participating institution. All patients provided written informed consent before enrolment. Women patients included in the study had to: 1 ) be ambulatory; 2) aged between 45 and 75 years; 3 ) be postmenopausal for at least 5 years; 4) have a body mass index between 18 and $30 \mathrm{~kg} / \mathrm{m}^{2} ; 5$ ) have a lumbar spine T-score (L2-L4) and/or femoral neck BMD lower than -2.0; and 6) be willing to provide written informed consent. Patients with the following criteria were excluded from the study: 1) disorders known to affect bone metabolism; 2) chronic diseases such as hyperthyroidism, hyperparathyroidism, osteomalacia, or diabetes; 3 ) treatment with bisphosphonates within the past year; 4) treatment with selective estrogen receptor modulators within the past 6 months; 5) treatment with activated vitamin D, calcitonin, estrogens, or androgens within the past 3 months; 6) treatment with warfarin; 7) chronic renal or liver disorders; and 8) history of malignant tumor. Patients were also excluded if one of these following laboratory tests was abnormal: 1) alkaline phosphatase (ALP) elevated $>10 \%$ of upper normal limit; 2) alanine aminotransferase (ALT) or aspartate aminotransferase (AST) elevated $>50 \%$ of upper normal limit; 3) serum creatinine levels $>133 \mu \mathrm{mmol} / \mathrm{L}$ (1.5 mg/dL); and 4) fasting blood glucose $>126 \mathrm{mg} / \mathrm{dL}$ (7.0 $\mathrm{mmol} / \mathrm{L})$.

\section{Study design}

This was a multicenter, randomized, double-blinded, doubledummy, noninferiority, positive drug-controlled clinical trial conducted in the following five investigational sites of the People's Republic of China: Department of Endocrinology, Peking Union Medical College Hospital, Beijing; Department of Obstetrics and Gynecology, General Hospital of the People's Liberation Army, Beijing; Metabolic Bone Disease and Genetic Research Unit, Department of Osteoporosis and Bone Disease, Shanghai Jiao Tong University Affiliated Sixth People's Hospital, Shanghai; Department of Geriatrics, Shanghai Huadong Hospital, Shanghai; and Department of Gynecology and Obstetrics, Beijing Hospital, Ministry of Public Health, Beijing.

No changes were made to the protocol during the study. Patients were randomized (using random number tables and sequentially numbered envelopes) in a $1: 1$ ratio to receive either menatetrenone (Eisai Co, Ltd, Tokyo, Japan) $15 \mathrm{mg}$, three times per day (45 mg/day) (Group M) or alfacalcidol (Haier Pharmaceutical Co, Ltd, Qingdao, People's Republic of China) $0.25 \mu \mathrm{g}$, twice per day $(0.5 \mu \mathrm{g} /$ day) (Group A) for 1 year. Additionally, patients in both groups received once daily Calcichew ${ }^{\circledR}$ (Nycomed Pharma, Zurich, Switzerland), containing $500 \mathrm{mg}$ of elemental calcium.

After screening and randomization (baseline), patients were followed up every 3 months. At each visit, occurrence and nature of the adverse effects were recorded for each patient. Blood levels of calcium and phosphorus, routine blood examination, urine routine examination, kidney function (creatinine), blood urea nitrogen, liver function tests (AST and ALT), and ALP were tested at months 0, 6, and 12. Biochemical markers of bone metabolism such as serum total OC and undercarboxylated OC (ucOC) were also measured at months 0,6 , and 12 .

Serum OC and ucOC were measured by a central laboratory (National Center for Clinical Laboratory, Beijing, People's Republic of China) using an electrochemiluminescence assay (Roche Diagnostics, Basel, Switzerland; 
intra-assay coefficient of variation $[\mathrm{CV}]$ of $2.2 \%$ and interassay CV of 5.9\%) and an enzyme-linked immunosorbent assay (Takara Bio, Otsu, Japan; intra-assay CV of $4.2 \%$ and inter-assay CV of $4.4 \%$ ).

BMDs were measured at the lumbar spine and hip by dual energy X-ray absorptiometry (phantom $\mathrm{CV}<1.5 \%$ ). Results at baseline and at months 6 and 12 were recorded. In order to obtain an optimal concordance between measurements from different centers, each imaging instrument was cross-calibrated using an external phantom (Synarc Inc, Newark, CA, USA). Calibration differences between individual scanners within a scanner family (ie, Lunar or Hologic) were adjusted using the results of the cross-calibration phantom. Systematic differences between the Lunar and Hologic scanners were adjusted using in vivo cross-calibration formulae from reported research studies. ${ }^{16,17}$ Lumbar and thoracic spine X-ray radiographs were taken at baseline and month 12. Morphometric vertebral fractures were defined as a decrease of $>20 \%$ in vertebral height (minimum, $4 \mathrm{~mm}$ ) by quantitative morphometry in radiographs. ${ }^{18}$

The primary endpoints were to evaluate the effects of treatment on BMD at the lumbar spine and hip. The secondary endpoints were to assess the effects of treatment on serum biochemical markers including serum $\mathrm{OC}$, ucOC, and ucOC/ OC ratio. The secondary endpoints also included the onset of new fractures and falls during observation.

\section{Statistical analysis}

The data analysis plan was finalized before database lock and unblinding. Analyses were carried out based on the intention-to-treat principle. Efficacy analysis was completed for the per-protocol set, including all patients who had undergone randomization, except for 23 patients who dropped out during the study (Group M: n=108; Group A: $n=105$ ). Safety analysis was performed for the safety set, and they included patients who had received at least one treatment dose, with the last available data in all subsequent measuring points (Group M: n=118; Group A: $\mathrm{n}=118$ ). All follow-up measurements were compared to the baseline values, and the change rates were calculated for each patient. Due to skewed distribution, the percentage change from the baseline of BMD was presented as the median. $P$-values of BMD compared with the baseline within each group were based on change from baseline. Differences within and between groups were tested by the Wilcoxon signed-rank test and Wilcoxon rank-sum test, respectively. Safety data were summarized for patients with adverse events for each group. Statistical analyses were performed using SAS v8 (SAS Institute Inc, Cary, NY, USA). A $P$-value $<0.05$ (two sides) was considered statistically significant.

\section{Results}

\section{Patients}

A total of 236 eligible women with postmenopausal osteoporosis were enrolled in the study, and they were randomly assigned to receive menatetrenone or alfacalcidol $(n=118$ each). Among them, 213 patients $(90.3 \%)$ completed the 1-year follow-up period, and 23 patients withdrew prematurely. In Group M, ten patients withdrew (adverse event $=4$, protocol violation $=1$, and nonmedical reason $=5$ ); and in Group A, 13 patients withdrew (adverse event $=8$, nonmedical reason $=5$ ).

\section{Baseline characteristics}

Patients' baseline characteristics are summarized in Table 1. There were no significant differences between the two groups for any variable. Also, there were no differences in blood pressure, routine blood analyses, routine urine analyses, and liver and kidney function tests between the two groups (data not shown).

\section{Changes in BMD}

Compared with baseline, BMD in Group $\mathrm{M}$ significantly increased by $1.2 \%$ and $2.7 \%$ at the lumbar spine and

Table I Baseline characteristics of the patients subjected to efficacy analysis

\begin{tabular}{|c|c|c|c|}
\hline & Menatetrenone & Alfacalcidol & $P$-value \\
\hline Number of patients & 108 & 105 & \\
\hline Age, years & $64.6 \pm 6.1$ & $64.2 \pm 6.3$ & 0.649 \\
\hline \multicolumn{4}{|l|}{ Years since menopause } \\
\hline age, years & $\mid 5.1 \pm 6.5$ & $15.6 \pm 6.6$ & 0.591 \\
\hline Height, cm & $154.2 \pm 5.4$ & $155.0 \pm 5.0$ & 0.267 \\
\hline Weight, kg & $55.2 \pm 7.2$ & $56.2 \pm 7.3$ & 0.322 \\
\hline \multicolumn{4}{|l|}{ Previous vertebral } \\
\hline fracture, number (\%) & $14(13.0)$ & $12(11.4)$ & 0.732 \\
\hline \multicolumn{4}{|c|}{ Bone mineral density, $\mathrm{mg} / \mathrm{cm}^{2}$} \\
\hline Lumbar spine & $805.0 \pm 115.1$ & $793.7 \pm 99.9$ & 0.654 \\
\hline Femoral neck & $644.6 \pm 101.1$ & $654.1 \pm 97.8$ & 0.374 \\
\hline Trochanter & $561.0 \pm 94.1$ & $567.8 \pm 81.5$ & 0.644 \\
\hline Serum calcium, mmol/L & $2.37 \pm 0.09$ & $2.37 \pm 0.11$ & 0.796 \\
\hline Serum phosphate, $\mathrm{mmol} / \mathrm{L}$ & $1.25 \pm 0.15$ & $1.23 \pm 0.18$ & 0.434 \\
\hline Serum total ALP, U/L & $75.5 \pm 19.4$ & $73.7 \pm 18.9$ & 0.479 \\
\hline Serum OC, ng/mL & $27.8 \pm 8.5$ & $26.1 \pm 8.5$ & 0.088 \\
\hline Serum ucOC, $\mathrm{ng} / \mathrm{mL}$ & $2.7 \pm 2.2$ & $2.4 \pm 1.9$ & 0.305 \\
\hline Serum ucOC/OC & $0.09 \pm 0.06$ & $0.09 \pm 0.07$ & 0.509 \\
\hline
\end{tabular}

Abbreviations: ALP, alkaline phosphatase; OC, osteocalcin; ucOC, undercarboxylated osteocalcin. 
trochanter, respectively, after 12 months of treatment $(P<0.001)$. In Group A, BMD significantly increased by $2.2 \%$ and $1.8 \%$ at the lumbar spine and trochanter, respectively $(P<0.001)$. There were no significant changes in the femoral neck BMD in both groups. There was no significant difference between the two groups after 6 and 12 months of treatment $(P>0.05)$ (Figure 1).

\section{Changes in $\mathrm{OC}$ and ucOC}

Figure 2 shows the effects of treatment on serum $\mathrm{OC}$ and ucOC levels and on the ucOC/OC ratio. In Group $\mathrm{M}$, after 12 months of treatment, $\mathrm{OC}$ and ucOC decreased by $38.7 \%$ and $82.3 \%$, compared with baseline $(P<0.001)$. In Group A, OC and ucOC also decreased by $25.8 \%$ and $34.8 \%$, respectively $(P<0.001)$. The decreases in serum $\mathrm{OC}$ and ucOC levels were more obvious in Group $M$ than
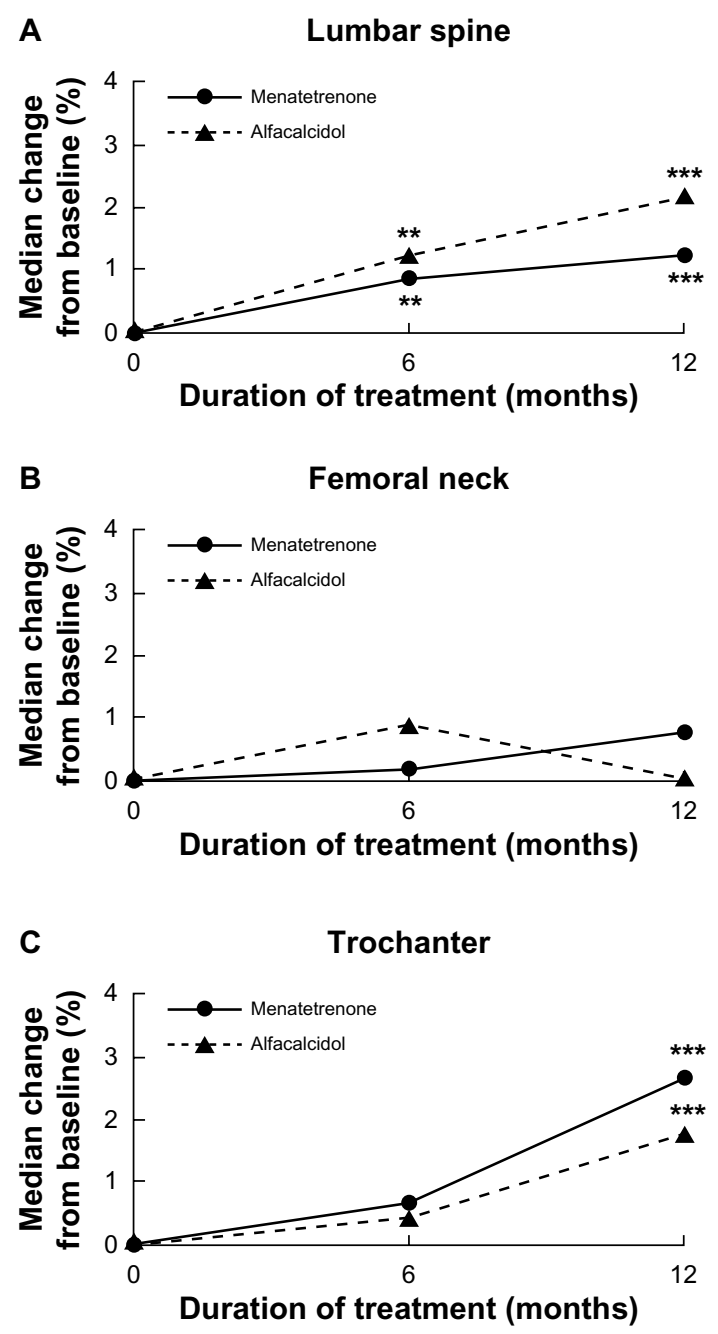

Figure I Median percentage of change over time in bone mineral density. Results are shown for bone mineral density at the (A) lumbar spine, (B) femoral neck, and (C) trochanter.

Notes: ${ }^{*} * P<0.01$; $* * * P<0.001$ compared to baseline. There were no differences between Group M and Group A.
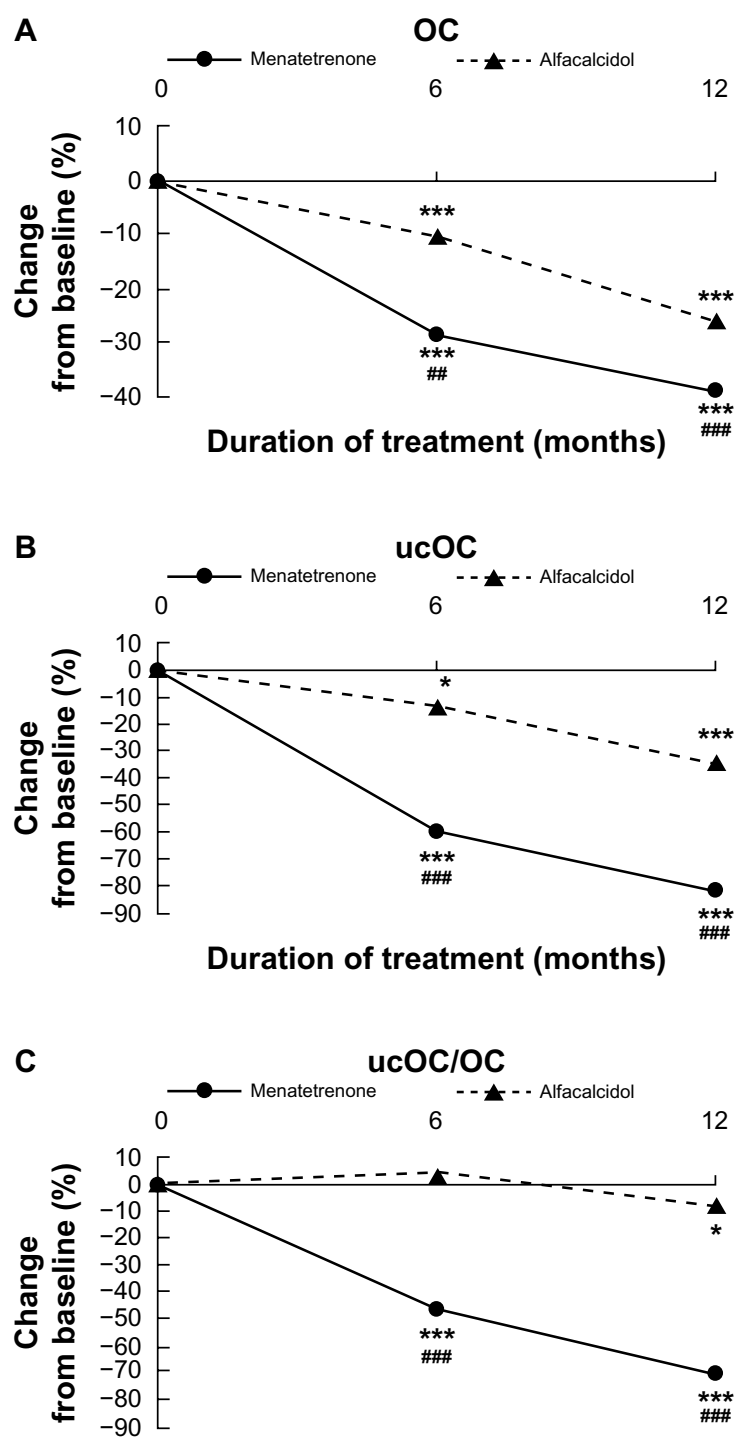

Duration of treatment (months)

Figure 2 Changes over time in (A) serum OC levels, (B) serum ucOC levels, and (C) ucOC/OC ratio.

Notes: ${ }^{*} P<0.05$; $* * * P<0.001$ compared to baseline; ${ }^{\# P} P<0.01$; ${ }^{\# P} P<0.00$ I for comparison between Group M and Group A.

Abbreviations: OC, osteocalcin; ucOC, undercarboxylated osteocalcin.

in Group A $(P<0.001)$. The ucOC/OC ratios also decreased after treatment, especially in Group M (Group M: $P<0.001$; Group A: $P<0.05$ ) (Figure 2).

\section{Changes in serum calcium, phosphorus, and ALP}

In Group $\mathrm{M}$, serum calcium was $2.37 \pm 0.09 \mathrm{mmol} / \mathrm{L}$, $2.42 \pm 0.12 \mathrm{mmol} / \mathrm{L}$, and $2.44 \pm 0.12 \mathrm{mmol} / \mathrm{L}$ at baseline, and after 6 and 12 months of treatment, respectively. Serum phosphorus was $1.25 \pm 0.15 \mathrm{mmol} / \mathrm{L}, 1.26 \pm 0.17 \mathrm{mmol} / \mathrm{L}$, and $1.27 \pm 0.18 \mathrm{mmol} / \mathrm{L}$ at baseline and after 6 and 12 months of treatment, respectively. Serum ALP was $75.5 \pm 19.4 \mathrm{U} / \mathrm{L}$, 
72.6 $\pm 24.0 \mathrm{U} / \mathrm{L}$, and $72.2 \pm 19.1 \mathrm{U} / \mathrm{L}$ at baseline, and after 6 and 12 months of treatment, respectively. There was no significant difference between the changes of serum calcium, phosphorus, and ALP before and after treatment (all $P>0.05$ ). In Group A, serum calcium was $2.37 \pm 0.11 \mathrm{mmol} / \mathrm{L}$, $2.43 \pm 0.14 \mathrm{mmol} / \mathrm{L}$, and $2.43 \pm 0.13 \mathrm{mmol} / \mathrm{L}$ at baseline and after 6 and 12 months of treatment, respectively. Serum phosphorus was $1.23 \pm 0.18 \mathrm{mmol} / \mathrm{L}, 1.28 \pm 0.17 \mathrm{mmol} / \mathrm{L}$, and $1.23 \pm 0.18 \mathrm{mmol} / \mathrm{L}$ at baseline, and after 6 and 12 months of treatment, respectively. Serum ALP was $73.7 \pm 18.9 \mathrm{U} / \mathrm{L}$, $73.0 \pm 18.6 \mathrm{U} / \mathrm{L}$, and $70.3 \pm 16.5 \mathrm{U} / \mathrm{L}$ at baseline, and after 6 and 12 months of treatment, respectively. There was no significant difference between the changes of serum calcium, phosphorus, and ALP before and after treatment (all $P>0.05$ ). There was no significant difference between the two groups after 6 and 12 months of treatment (all $P>0.05$ ).

\section{New bone fractures and falls}

Two patients $(1.85 \%, 2 / 108)$ in Group M suffered new bone fractures during the 1-year observation period, which included one tibia fracture and one femoral neck fracture. In Group A, four patients $(3.81 \%, 4 / 105)$ suffered new fractures, which included three lumbar spine compressive fractures and one forearm fracture. Thirteen patients $(12.04 \%, 13 / 108)$ in Group $\mathrm{M}$ experienced new falls during the 1-year observation period, while ten patients $(9.52 \%, 10 / 105)$ in Group A experienced new falls. There were no significant differences in new bone fractures and falls between the two groups $(P>0.05)$.

\section{Safety analysis}

The safety profile was similar between menatetrenone and alfacalcidol. The incidence of related adverse events was 7.7\% in Group $\mathrm{M}$ and 5.1\% in Group A. Related adverse events reported during the study are shown in Table 2. Four patients in Group $\mathrm{M}$ and eight patients in Group A dropped

Table 2 Related adverse events during the study

\begin{tabular}{|c|c|c|}
\hline Related side effects & $\begin{array}{l}\text { Group M } \\
(n=|| 8)\end{array}$ & $\begin{array}{l}\text { Group A } \\
(n=\mid 18)\end{array}$ \\
\hline Constipation & $2(1.7 I \%)$ & 0 \\
\hline Abdominal pain & I $(0.85 \%)$ & $5(4.24 \%)$ \\
\hline Abdominal distension & $2(1.7 \mid \%)$ & $2(1.69 \%)$ \\
\hline Esophagus burning & I (0.85\%) & 0 \\
\hline Dysfunction of stomach intestine & $2(1.7 \mid \%)$ & I (0.85\%) \\
\hline Gastritis & I (0.85\%) & 0 \\
\hline Belching & $\mathrm{I}(0.85 \%)$ & 0 \\
\hline AST/ALT elevated & $2(1.7 \mid \%)$ & 0 \\
\hline Hypercalcemia & I $(0.85 \%)$ & 0 \\
\hline Leukocytopenia & I (0.85\%) & 0 \\
\hline
\end{tabular}

Abbreviations: ALT, alanine aminotransferase; AST, aspartate aminotransferase. out of the study due to adverse events. The majority of these adverse events were gastrointestinal symptoms and were of mild to moderate intensity. Serum ALT or/and AST were mildly elevated $(73 \mathrm{U} / \mathrm{L}, 155 \mathrm{U} / \mathrm{L})$ in two patients in Group $\mathrm{M}$ at month 12. However, their liver function returned to within the normal range after 2 and 8 months, respectively. Transient hypercalcemia and leukocytopenia were observed in Group M. Unrelated adverse events included bone pain $(1.7 \%$ in Group $\mathrm{M}$ and $0.9 \%$ in Group A) and joint pain (6.8\% in Group $\mathrm{M}$ and 3.4\% in Group A), which were mild and showed no difference between the two groups. No other clinically significant unexpected adverse events were reported in either group during the study.

\section{Discussion}

The present study investigated the efficacy and safety of menatetrenone in Chinese postmenopausal osteoporotic women. A significant increase in BMD by $1.2 \%$ and $2.7 \%$ at the lumbar spine and trochanter, respectively, from baseline and a significant decrease in $\mathrm{OC}$ and ucOC by $38.7 \%$ and $82.3 \%$, respectively, from baseline were observed with menatetrenone treatment. Moreover, the safety profile of menatetrenone was similar to alfacalcidol. Being the first clinical trial to investigate the effects of menatetrenone on a Chinese population, the study results could provide a valid clinical basis on treating osteoporosis.

Since the study involved postmenopausal osteoporotic women (BMD T-score less than -2.0), placebo control was not used due to ethical reasons. Alfacalcidol is a recognized drug in treating osteoporosis in the People's Republic of China. Studies in Japan showed no differences in BMD changes between menatetrenone and alfacalcidol in postmenopausal women. ${ }^{19}$ Therefore, alfacalcidol was chosen as a positive drug control to demonstrate the noninferiority of these two drugs. Results showed that menatetrenone $45 \mathrm{mg} /$ day could significantly improve lumbar spine and trochanter BMD after 12 months of treatment. The dose of menatetrenone ( $45 \mathrm{mg} /$ day) used in this study is the standard dose to treat osteoporosis. As per the primary osteoporosis diagnosis and treatment guidelines of the Chinese Society of Osteoporosis and Bone Mineral Research, the dose of alfacalcidol in treating osteoporosis is $0.5-1.0 \mu \mathrm{g} /$ day. ${ }^{20}$ In order to avoid the side effect of high dose alfacalcidol such as hypercalcemia and hypercalciuria, ${ }^{21}$ a low dose of $0.5 \mu \mathrm{g} /$ day alfacalcidol was used in this study. No differences were observed between menatetrenone and alfacalcidol $(1.2 \%$ versus $2.2 \%$ in the lumbar spine, and $2.7 \%$ versus $1.8 \%$ in the trochanter, respectively) with respect to changes in BMD. However, menatetrenone had more effect on OC and ucOC than alfacalcidol. 
Some randomized controlled trials showed that menatetrenone had only a modest impact or no impact on BMD. ${ }^{22-24}$ In the study by Orimo et al, ${ }^{19}$ there were no differences observed in BMD between menatetrenone $45 \mathrm{mg} /$ day and alfacalcidol $1 \mu \mathrm{g}$ /day in 24 and 48 weeks. Although menatetrenone has only a modest effect on bone loss, it may have the potential to prevent osteoporotic fractures. Vitamin K deficiency has been reported to contribute to the occurrence of fractures in elderly women, especially hip fractures, ${ }^{25-28}$ and it is also a strong predictor of vertebral fractures in patients on hemodialysis. ${ }^{29} \mathrm{~A}$ randomized controlled trial suggested that vitamin K2 sustained lumbar $\mathrm{BMD}$ and reduced the incidence of vertebral fractures, with a reported reduction of $53 \%$ in patients with postmenopausal osteoporosis; this rate was similar to the rate reported with treatment with bisphosphonates. ${ }^{22}$ Knapen et al ${ }^{24}$ indicated that menatetrenone improved the bone strength of the femoral neck by improving femoral neck width and maintaining the indices of compression, bending, and impact strength. These could lead to a decreased incidence of clinical fractures. In the present study, $1.9 \%$ of patients experienced a new fracture in Group M during 1 year of treatment, while this proportion was 3.8\% in Group A; however, there was no significant difference between the two groups. There was also no difference in new falls between the two groups.

Menatetrenone is thought to be involved in bone formation as an essential cofactor for $\gamma$-carboxylation of OC. Rapid conversion of ucOC to carboxylated OC following menatetrenone treatment was confirmed in elderly osteoporotic women with vertebral fractures. ${ }^{30-33}$ The present study's results show that OC and ucOC decreased by $38.7 \%$ and $82.3 \%$, respectively, compared with baseline. The ucOC/OC ratio also decreased. Another study showed that menatetrenone reduced serum ucOC levels, and a study even showed a decrease in serum ucOC levels after 1 month of therapy. ${ }^{34}$ However, the results on serum total OC levels are inconsistent. Some studies showed an increase in serum total OC levels, ${ }^{22,24}$ while another showed a decrease in serum total OC levels by vitamin K2 supplementation. ${ }^{35}$ Two explanations can be proposed for this discrepancy: 1) since more OC becomes carboxylated and functional with the improvement of vitamin K2 status, a lesser amount is synthesized and released into circulation; and 2) more functional $\mathrm{OC}$ is bound into bones rather than circulating in the blood stream, thus lowering serum total OC levels. ${ }^{35,36}$ Studies have showed that ucOC, but not total OC, is a marker predicting hip fracture in elderly women. ${ }^{25-27}$

Menatetrenone tolerability was also evaluated in this study. The safety profile of menatetrenone was similar to alfacalcidol. Adverse events included gastrointestinal symptoms, mild and transient hypercalcemia, leukocytopenia, and abnormalities in liver function. No severe side effects were ever reported before for menatetrenone. ${ }^{32,36}$ Even in this study, menatetrenone was well tolerated by Chinese postmenopausal women. Moreover, the treatment with menatetrenone is a pharmacoeconomically viable option.

There are some limitations to this study. The sample size was not large enough, and trial duration was not long enough to evaluate the risk of fracture as the primary endpoint. However, results did show important short-term effects in BMD, OC, and ucOC levels, suggesting that these short-term changes should have an impact on the long-term risk of fractures. Also, a food frequency questionnaire was not performed to calculate vitamin $\mathrm{K}$ intake. Biochemical markers reflecting bone turnover were not measured. However, changes in BMD directly reflected the balance between bone resorption and bone deposition, and the study results showed that menatetrenone supplementation had a positive impact on BMD.

\section{Conclusion}

After 1 year of treatment with menatetrenone, BMD at the lumbar spine and hip were significantly increased in Chinese postmenopausal osteoporotic women, and that effect was comparable to alfacalcidol. These positive changes in BMD were reflected by changes in markers of bone metabolism, and menatetrenone had more effects on these markers. Menatetrenone was well tolerated and safe in the study population. Thus, menatetrenone is an effective and safe choice for the treatment of postmenopausal osteoporosis in Chinese women.

\section{Acknowledgments}

This study was financially supported by Eisai China Inc. The authors thank Haier Pharmaceutical Co, Ltd, which supported this study by supplying alfacalcidol. The authors thank DMS for completing the statistical analysis of the results.

\section{Disclosure}

The authors report no conflicts of interest in this work.

\section{References}

1. NIH Consensus Development Panel on Osteoporosis Prevention, Diagnosis, and Therapy. Osteoporosis prevention, diagnosis, and therapy. JAMA. 2001;285(6):785-795.

2. Raisz LG. Pathogenesis of osteoporosis: concepts, conflicts, and prospects. J Clin Invest. 2005;115(12):3318-3325

3. Liberman UA, Weiss SR, Broll J, et al. Effect of oral alendronate on bone mineral density and the incidence of fractures in postmenopausal osteoporosis. The Alendronate Phase III Osteoporosis Treatment Study Group. N Engl J Med. 1995;333(22):1437-1443. 
4. Barrett-Connor E, Mosca L, Collins P, et al. Effects of raloxifene on cardiovascular events and breast cancer in postmenopausal women. N Engl J Med. 2006;355(2):125-137.

5. Rossouw JE, Anderson GL, Prentice RL, et al. Risks and benefits of estrogen plus progestin in healthy postmenopausal women: principal results from the Women's Health Initiative randomized controlled trial. JAMA. 2002;288(3):321-333.

6. Cummings SR, San Martin J, McClung MR, et al. Denosumab for prevention of fractures in postmenopausal women with osteoporosis N Engl J Med. 2009;361(8):756-765.

7. Hauschka PV, Lian JB, Cole DE, Gundberg CM. Osteocalcin and matrix Gla protein: vitamin K-dependent proteins in bone. Physiol Rev. 1989;69(3):990-1047.

8. Koshihara Y, Hoshi K. Vitamin K2 enhances osteocalcin accumulation in the extracellular matrix of human osteoblasts in vitro. J Bone Miner Res. 1997;12(3):431-438.

9. Shearer MJ. Vitamin K. Lancet. 1995;345(8944):229-234.

10. Feskanich D, Weber P, Willett WC, Rockett H, Booth SL, Colditz GA. Vitamin $\mathrm{K}$ intake and hip fractures in women: a prospective study. Am J Clin Nutr. 1999;69(1):74-79.

11. Booth SL, Tucker KL, Chen H, et al. Dietary vitamin K intakes are associated with hip fracture but not with bone mineral density in elderly men and women. Am J Clin Nutr. 2000;71(5):1201-1208.

12. Sato Y, Honda Y, Hayashida N, Iwamoto J, Kanoko T, Satoh K. Vitamin K deficiency and osteopenia in elderly women with Alzheimer's disease. Arch Phys Med Rehabil. 2005;86(3):576-581.

13. Kaneki M, Hodges SJ, Hosoi T, et al. Japanese fermented soybean food as the major determinant of the large geographic difference in circulating levels of vitamin K2: possible implications for hip-fracture risk. Nutrition. 2001;17(4):315-321.

14. Cockayne S, Adamson J, Lanham-New S, Shearer MJ, Gilbody S, Torgerson DJ. Vitamin K and the prevention of fractures: systematic review and meta-analysis of randomized controlled trials. Arch Intern Med. 2006;166(12):1256-1261.

15. Iwamoto J, Takeda T, Sato Y. Role of vitamin K2 in the treatment of postmenopausal osteoporosis. Curr Drug Saf. 2006;1(1):87-97.

16. Hui SL, Gao S, Zhou XH, et al. Universal standardization of bone density measurements: a method with optimal properties for calibration among several instruments. J Bone Miner Res. 1997;12(9):1463-1470.

17. Lu Y, Fuerst T, Hui S, Genant HK. Standardization of bone mineral density at femoral neck, trochanter and Ward's triangle. Osteoporos Int. 2001;12(6):438-444.

18. Genant HK, Wu CY, van Kuijk C, Nevitt MC. Vertebral fracture assessment using a semiquantitative technique. J Bone Miner Res. 1993;8(9): 1137-1148.

19. Orimo H, Fujita T, Onomura T, Inoue T, Kushida K, Shiraki M. Clinical evaluation of Ea-0167 (menatetrenone) in the treatment of osteoporosis. Rinsho Hyoka (Clinical Evaluation). 1992;20(1):45-100.

20. Osteoporosis and Bone Mineral Society of the Chinese Medical Association. Primary osteoporosis diagnosis and treatment guidelines. Chinese Journal of Osteoporosis and Bone Mineral Research. 2011;4:2-17.

21. O’Donnell S, Moher D, Thomas K, Hanley DA, Cranney A. Systematic review of the benefits and harms of calcitriol and alfacalcidol for fractures and falls. J Bone Miner Metab. 2008;26(6):531-542.
22. Shiraki M, Shiraki Y, Aoki C, Miura M. Vitamin K2 (menatetrenone) effectively prevents fractures and sustains lumbar bone mineral density in osteoporosis. J Bone Miner Res. 2000;15(3):515-521.

23. Inoue $\mathrm{T}$, Fujita $\mathrm{T}$, Kishimoto $\mathrm{H}$, et al. Randomized controlled study on the prevention of osteoporotic fractures (OF study): a Phase IV clinical study of $15 \mathrm{mg}$ menatetrenone capsules. J Bone Miner Metab. 2009;27(1):66-75

24. Knapen MH, Schurgers LJ, Vermeer C. Vitamin K2 supplementation improves hip bone geometry and bone strength indices in postmenopausal women. Osteoporos Int. 2007;18(7):963-972.

25. Szulc P, Chapuy MC, Meunier PJ, Delmas PD. Serum undercarboxylated osteocalcin is a marker of the risk of hip fracture in elderly women. J Clin Invest. 1993;91(4):1769-1774.

26. Szulc P, Chapuy MC, Meunier PJ, Delmas PD. Serum undercarboxylated osteocalcin is a marker of the risk of hip fracture: a three year follow-up study. Bone. 1996;18(5):487-488.

27. Vergnaud P, Garnero P, Meunier PJ, Breart G, Kamihagi K, Delmas PD. Undercarboxylated osteocalcin measured with a specific immunoassay predicts hip fracture in elderly women: the EPIDOS Study. J Clin Endocrinol Metab. 1997;82(3):719-724.

28. Luukinen H, Kakonen SM, Pettersson K, et al. Strong prediction of fractures among older adults by the ratio of carboxylated to total serum osteocalcin. J Bone Miner Res. 2000;15(12):2473-2478.

29. Fusaro M, Noale M, Viola V, et al. Vitamin K, vertebral fractures, vascular calcifications, and mortality: VItamin K Italian (VIKI) dialysis study. J Bone Miner Res. 2012;27(11):2271-2278.

30. Iwamoto J, Takeda T, Ichimura S. Effect of combined administration of vitamin D3 and vitamin K2 on bone mineral density of the lumbar spine in postmenopausal women with osteoporosis. J Orthop Sci. 2000;5(6):546-551.

31. Ozuru R, Sugimoto T, Yamaguchi T, Chihara K. Time-dependent effects of vitamin K2 (menatetrenone) on bone metabolism in postmenopausal women. Endocr J. 2002;49(3):363-370.

32. Ushiroyama T, Ikeda A, Ueki M. Effect of continuous combined therapy with vitamin $\mathrm{K}(2)$ and vitamin $\mathrm{D}(3)$ on bone mineral density and coagulofibrinolysis function in postmenopausal women. Maturitas. 2002;41(3):211-221.

33. Miki T, Nakatsuka K, Naka H, et al. Vitamin K(2) (menaquinone 4) reduces serum undercarboxylated osteocalcin level as early as 2 weeks in elderly women with established osteoporosis. J Bone Miner Metab. 2003;21(3):161-165.

34. Shiraki M, Itabashi A. Short-term menatetrenone therapy increases gamma-carboxylation of osteocalcin with a moderate increase of bone turnover in postmenopausal osteoporosis: a randomized prospective study. J Bone Miner Metab. 2009;27(3):333-340.

35. Cheung AM, Tile L, Lee Y, et al. Vitamin K supplementation in postmenopausal women with osteopenia (ECKO trial): a randomized controlled trial. PLoS Med. 2008;5(10):e196.

36. Iwamoto J, Sato Y, Takeda T, Matsumoto H. High-dose vitamin K supplementation reduces fracture incidence in postmenopausal women: a review of the literature. Nutr Res. 2009;29(4):221-228.
Clinical Interventions in Aging

\section{Publish your work in this journal}

Clinical Interventions in Aging is an international, peer-reviewed journal focusing on evidence-based reports on the value or lack thereof of treatments intended to prevent or delay the onset of maladaptive correlates of aging in human beings. This journal is indexed on PubMed Central, MedLine, the American Chemical Society's 'Chemical Abstracts

\section{Dovepress}

Service' (CAS), Scopus and the Elsevier Bibliographic databases. The manuscript management system is completely online and includes a very quick and fair peer-review system, which is all easy to use. Visit http://www.dovepress.com/testimonials.php to read real quotes from published authors. 